\title{
Avaliação do processamento auditivo em crianças com dificuldades de aprendizagem
}

\section{Auditory processing evaluation in children with learning difficulties}

\author{
Lucilene Engelmann ${ }^{1}$, Maria Inês Dornelles da Costa Ferreira²
}

\begin{abstract}
RESUMO
Objetivo: Esclarecer a relação entre dificuldades de aprendizagem e o transtorno do processamento auditivo em uma turma de segunda série. Métodos: Através da aplicação de testes de leitura os alunos foram classificados quanto à fluência em leitura, sendo um com maior fluência (grupo A) e outro com menor fluência (grupo B). Os testes de processamento auditivo foram comparados entre os grupos. Resultados: Todos os participantes apresentaram dificuldades de aprendizagem e transtorno do processamento auditivo em quase todos os subperfis primários. Verificou-se que a variável memória sequencial verbal do grupo de menor fluência em leitura (grupo B) foi significantemente melhor ( $\mathrm{p}=0,030)$. Conclusão: Questiona-se o diagnóstico de transtorno primário do processamento auditivo e salienta-se a importância da memória sequencial verbal no aprendizado da leitura e escrita. Em face do que foi observado, mais pesquisas deverão ser realizadas objetivando o estudo dessa variável e sua relação com o processamento auditivo temporal.
\end{abstract}

Descritores: Percepção auditiva; Transtornos da percepção auditiva; Criança; Leitura; Escrita; Aprendizagem; Deficiências do desenvolvimento; Transtornos da audição

\section{INTRODUÇÃO}

O processamento auditivo é definido como um conjunto de operações que o sistema auditivo realiza: receber, detectar, atender, reconhecer, associar e integrar os estímulos acústicos para posteriormente programar uma resposta ${ }^{(1)}$, ou seja, é um conjunto de habilidades necessárias para analisar e interpretar os padrões sonoros ${ }^{(2)}$.

A via auditiva periférica encontra-se pronta ao nascimento e engloba a orelha externa, orelha média e orelha interna. A cóclea, que fica localizada na orelha interna, representa o componente sensorial que transforma o impulso sonoro em elétrico para que o componente neural receba, analise e programe uma resposta ${ }^{(3)}$. Este se desenvolve a partir de experiências sonoras pelas quais a criança passa nos primeiros anos de vida, período em que se formam as primeiras conexões neuronais que ocorrem entre o nascimento e os quatro anos de idade ${ }^{(4)}$. Outros estudos apontam a existência de um período de alta plasticidade auditiva até os seis anos de idade ${ }^{(5-6)}$.

Trabalho de conclusão do Curso de Fonoaudiologia realizado no Laboratório de Audiologia do Centro Universitário Metodista IPA - Porto Alegre (RS), Brasil.

(1) Fonoaudióloga clínica - Porto Alegre (RS) - Brasil

(2) Doutora, Docente do Curso de Fonoaudiologia do Centro Universitário Metodista IPA - Porto Alegre (RS), Brasil e da Faculdade Nossa Senhora de Fátima - Caxias do Sul (RS), Brasil.

Endereço para correspondência: Maria Inês Dornelles da Costa Ferreira. R. Luiz Afonso, 158/702, Cidade Baixa, Porto Alegre - RS, CEP 90050-310.

E-mail: costa.ferreira@terra.com.br

Recebido em: 7/2/2008; Aceito em: 28/9/2008
Um estudo confirmou que a maturação neural interferiu de maneira significativa no desempenho dos testes comportamentais do processamento auditivo, entre as idades de oito e dez $\operatorname{anos}^{(7)}$.

Nesta idade as crianças já estão aptas a responderem aos testes comportamentais, podendo apresentar melhora no desempenho na faixa dos dez anos ${ }^{(7)}$.

As habilidades auditivas avaliadas através dos testes comportamentais são: detecção, localização da fonte sonora, discriminação auditiva, atenção seletiva, figura-fundo auditiva, fechamento auditivo, separação e integração binaural e memória sequencial auditiva. A avaliação do processamento auditivo classifica-o como normal ou alterado. Dessa forma, o transtorno do processamento auditivo (TPA) é uma alteração da audição na qual há um impedimento da habilidade de analisar e/ou interpretar padrões sonoros ${ }^{(2)}$. Tal transtorno pode ser associado às dificuldades de aprendizagem $(\mathrm{DA})^{(8-9)}$.

Assim, os indivíduos com queixas de dificuldades escolares geralmente apresentam pior desempenho em testes de processamento auditivo em função do atraso na maturação das habilidades auditivas. Tais habilidades são fundamentais para o processo de aprendizagem da leitura e da escrita ${ }^{(7)}$.

O termo dificuldades de aprendizagem não se refere a um único distúrbio, mas a uma ampla gama de problemas que podem afetar qualquer área do desempenho acadêmico ${ }^{(10)} \mathrm{e}$ são problemas que podem alterar as possibilidades da criança de aprender independente de suas condições neurológicas para fazê-1o ${ }^{(11)}$. 
As dificuldades de aprendizagem podem ser classificadas como naturais ou secundárias. As naturais são aquelas em que as causas estão relacionadas a fatores como a escola (proposta pedagógica), pouca assiduidade da criança e aspectos referentes à família. Geralmente essas dificuldades são transitórias e tendem a ser superadas ${ }^{(10-12)}$.

As dificuldades secundárias são aquelas decorrentes de outras patologias como: portadores de déficits cognitivos, sensoriais e quadros neurológicos mais graves ${ }^{(11)}$.

As características mais encontradas na grafia de crianças com dificuldades de aprendizagem são: erros ortográficos (omissões, inversões, substituições, transposições, erros no conversor fonema grafema), lentidão ao realizar a cópia, alterações no traçado da letra e uso incorreto do espaço. A leitura é lenta acarretando baixa compreensão e confusão com palavras semelhantes ${ }^{(12)}$. Tais crianças também apresentam recusa em falar sobre a escola e queixas referentes ao trabalho escolar $^{(10)}$.

A avaliação do processamento auditivo nos permite classificar o TPA em cinco subperfis, sendo três primários e dois secundários a partir da avaliação simplificada e dos testes comportamentais. Os subperfis primários são: decodificação auditiva, prosódia ou função não verbal e integração. Os secundários são: associação auditiva e organização de saí$\mathrm{da}^{(13-14)}$. Neste trabalho serão analisados somente os subperfis primários.

O subperfil de decodificação é considerado o subperfil mais específico à modalidade auditiva, sendo considerado por alguns como o verdadeiro TPA ${ }^{(13-14)}$. Crianças com alteração neste subperfil apresentam dificuldades para discriminar os sons ouvidos, analisar os sons da fala, dificuldade para ouvir em ambientes ruidosos, vocabulário restrito e substituições de grafemas na escrita. As habilidades auditivas mais alteradas no déficit decodificação são fechamento auditivo, discriminação auditiva, separação e integração binaural e processamento temporal ${ }^{(2,13-14)}$.

Os achados típicos na avaliação do processamento auditivo são: déficit de orelha direita ou bilateral nos testes de dicóticos, baixo desempenho em testes monoaurais de baixa redundância e limiares elevados de detecção de intervalos de silêncio e a análise qualitativa dos tipos de erros evidencia omissões e substituições fonêmicas ${ }^{(13-14)}$.

O subperfil de integração pode ser resultante de uma alteração inter-hemisférica ocasionando apresentando dificuldades em integrar os estímulos auditivos com os visuais ou táteis. Neste caso, as crianças podem apresentar alterações na leitura, escrita, ritmo e pausas. Nos testes específicos encontram-se alterações na nomeação de padrões temporais com melhora da resposta na imitação acompanhada de desempenho rebaixado na orelha esquerda nos testes dicóticos verbais. Na análise qualitativa, os indivíduos podem apresentar omissões de palavras em escuta dicótica e lentidão nas respostas ${ }^{(14)}$.

As dificuldades mais encontradas em sujeitos com alteração no subperfil de prosódia ou função não verbal são: dificuldades em utilizar e identificar as características supra-segmentais de um enunciado, compreender piadas, reproduzir melodias e emitir palavras que exprimam sentimentos ${ }^{(1)}$. Nos resultados dos testes comportamentais podem ser encontradas alterações na nomeação e imitação de padrões temporais e rebaixamento na orelha esquerda em testes dicóticos verbais ${ }^{(14-15)}$.

São comportamentos característicos dos indivíduos com TPA alterações na comunicação oral ou na utilização de regras gramaticais, inversões de grafemas, alterações da noção de lateralidade, agitação, hiperatividade ou apatia, memória auditiva prejudicada e dificuldade em compreender a mensagem acústica em ambientes ruidosos. Desta forma, o objetivo do presente estudo é esclarecer a relação entre as dificuldades de aprendizagem e o transtorno do processamento auditivo (TPA), contribuindo na ampliação da literatura sobre a avaliação do processamento auditivo em crianças com dificuldades de aprendizagem, e demonstrando a importância do trabalho fonoaudiológico nas escolas orientando pais e professores quanto aos procedimentos nesses $\operatorname{casos}^{(1,13-14)}$.

\section{MÉTODOS}

O presente estudo foi aprovado pelo Comitê de Ética da instituição de origem sob o número 064/2007.

Este estudo caracteriza-se por ser um estudo de campo, de caráter exploratório, transversal, individual e contemporâneo, cujos dados são relatados de forma qualitativa e quantitativa. Os fatores em estudo foram TPA, leitura e escrita, e o desfecho foi à relação entre eles.

Participaram da pesquisa 30 crianças, alunos regularmente matriculados na $2^{\mathrm{a}}$ série do Ensino Fundamental de uma escola pública de nível socioeconômico baixo da cidade de Porto Alegre. Desses, 16 eram do gênero masculino $(53,3 \%)$ e 14 do feminino $(46,7 \%)$, com idades variando entre sete e 11 anos.

A segunda série foi escolhida em função da conclusão do processo de alfabetização e por ser uma faixa etária em que as dificuldades na aprendizagem começam a ser observadas pelos pais e professores. A turma foi indicada pela escola.

A diretora da escola assinou o termo de autorização institucional, e os pais e/ou responsáveis, o Termo de Consentimento Livre e Esclarecido.

Para participar da pesquisa, as crianças deveriam apresentar audição periférica normal e curva timpanométrica tipo $\mathrm{A}^{(16)}$; e cursar a segunda série do Ensino Fundamental.

Das 30 crianças que integravam a $2^{\mathrm{a}}$ série, nove foram excluídas do estudo: uma por falta de assinatura do termo de consentimento livre e esclarecido e oito por apresentar alterações na avaliação audiológica básica, (sete apresentaram alterações na audiometria tonal e uma na curva timpanométrica). Tais participantes foram encaminhados ao médico otorrinolaringologista para tratamento.

Dessa forma, durante os meses de agosto e setembro de 2007, 21 crianças foram submetidas às avaliações de processamento auditivo, avaliação da escrita e avaliação da leitura.

Os instrumentos utilizados para selecionar os participantes foram: audiometria tonal e vocal, realizada em cabina acústica, e medidas de imitância acústica para avaliar a audição periférica. Ambos permitem a classificação da audição periférica como normal ou alterada.

Para a audiometria tonal foram utilizados os audiômetros AC30 e AC33 da marca Interacoustics $®$, e para as medidas de imitância acústica foi utilizado o imitanciômetro AT 22t, da 
marca Interacoustics ${ }^{\circledR}$. Todos os equipamentos encontravamse devidamente calibrados.

Uma parte da coleta dos dados foi realizada na própria escola, em uma sala cedida pela doordenação. O ditado balanceado foi realizado com toda a turma e as avaliações da fluência em leitura e da compreensão leitora foram realizadas individualmente. Para a aplicação do ditado balanceado foi entregue uma folha branca e ditado as palavras para que os participantes escrevessem. Caso errassem eram orientados a não apagar e escrever ao lado a palavra correta.

$\mathrm{Na}$ avaliação da leitura silenciosa foram observados o tempo empregado e a utilização de apoio articulatório. A avaliação da leitura oral foi realizada com o mesmo texto utilizado na leitura silenciosa em que foram observados: fluência, tempo empregado, volume, pontuação, omissão ou acréscimo de palavras e/ou fonemas, repetição de palavra ou frases e substituição de palavras ou fonemas ${ }^{(17)}$.

Para a avaliação da escrita foi utilizado um ditado balanceado e, para avaliar a fluência e a compreensão leitora foi utilizado o texto "A coisa" acompanhado de dez questões sobre o mesmo ${ }^{(18)}$.

Através dos testes de leitura e escrita a intenção das pesquisadoras era classificar as crianças em dois grupos; um com dificuldades na aprendizagem e outro sem dificuldades de aprendizagem, para que os resultados dos testes de processamento auditivo fossem analisados através da comparação entre os grupos.

Após a realização de tais testes verificou-se que os mesmos foram muito alterados. Convém ressaltar, que os alunos repetentes não foram excluídos da amostra. Diante disso os participantes foram reagrupados de acordo com o número de palavras lidas por minuto (até 40 palavras por minuto) e com o tempo da leitura silenciosa e oral (até cinco minutos).

No primeiro grupo (grupo A) os participantes leram mais de 40 palavras por minuto e concluíram a leitura silenciosa e oral em até cinco minutos. Na leitura oral respeitaram a pontuação, não omitiram palavras, não acrescentaram sílabas e palavras e não as repetiram. Este grupo foi composto por nove dos participantes.

As outras 12 crianças leram menos de 40 palavras por minuto e finalizaram a leitura oral e silenciosa após cinco minutos e, por este motivo foram classificadas no segundo grupo (grupo B).

O teste de compreensão leitora e o ditado balanceado mencionados anteriormente, não fizeram parte da distinção entre os grupos por mostrarem-se invariáveis, ou seja, alguns participantes do grupo A com maior fluência em leitura não tiveram bom desempenho nos testes de compreensão leitora e/ou ditado balanceado. Da mesma forma, alguns participantes do grupo $\mathrm{B}$, com menor fluência em leitura obtiveram resultados superiores ao grupo A em compreensão leitora e/ ou ditado balanceado. Maiores detalhes deste aspecto serão abordados na discussão.

Os instrumentos utilizados para avaliar os participantes no que se refere ao processamento auditivo foram: instrumentos musicais (sino, guizo, agogô e coco) para a realização da avaliação simplificada. Posteriormente os testes comportamentais de processamento auditivo foram realizados em cabina acústica com o audiômetro de dois canais, modelo AC30 (Interacoustics $\left.{ }^{\circledR}\right)$ acoplado ao CD player da marca Sony ${ }^{\circledR}$. Tais testes são comercializados em $\mathrm{CD}^{(19-20)}$.

O primeiro teste realizado foi o dicótico de dígitos - DD seguido do SSW. Na sequência utilizou-se o teste Pitch Pattern Sequence - PPS nas condições murmurando e nomeando.

Os testes acima citados foram escolhidos devido aos objetivos da pesquisa em relação à amostra selecionada e por oferecerem mais elementos para o diagnóstico da função auditiva central quando comparados aos demais testes.

Este estudo associou o resultado dos testes de processamento auditivo com idade e gênero.

Para análise estatística foi utilizado o programa The SAS System for Windows ${ }^{\circledR}$ (Statistical Analysis System), versão 8.02. O nível de significância adotado para os testes estatísticos foi de $5 \%$.

Para analisar a relação entre as variáveis categóricas foi utilizado o teste Qui-quadrado ou, quando necessário, o teste exato de Fisher (presença de valores esperados menores que cinco). Para comparar as variáveis numéricas entre os grupos de uma variável categórica foi utilizado o teste de MannWhitney (para dois grupos). Já para analisar a relação entre as variáveis numéricas foi utilizado o coeficiente de correlação de Spearman. Ao descrever o perfil da amostra segundo as variáveis em estudo, foram realizadas tabelas de frequência das variáveis categóricas, com valores de frequência absoluta (n) e percentual (\%), e estatísticas descritivas das variáveis contínuas, com valores de média, desvio padrão, valores mínimo e máximo, mediana e quartis.

\section{RESULTADOS}

A população constitui-se de uma turma de $2^{\text {a }}$ série, totalizando 21 crianças, observando-se os seguintes percentuais: sete meninas $(33,3 \%)$ e 14 meninos $(66,7 \%)$.

Quanto às idades dos participantes, três têm sete anos $(14,3 \%), 11$ têm oito $(52,4 \%)$, cinco têm nove anos $(23,9 \%)$ e dois participantes têm 11 anos $(9,4 \%)$. A turma foi indicada pela escola, visto as dificuldades apresentadas por esses alunos.

A Tabela 1 permite a visualização das ocorrências de alterações dos subperfis do PA. Os subperfis de decodificação e integração ocorreram em 19 participantes totalizando (90,5\%), e o subperfil denominado prosódia ocorreu em 18 crianças $(85,7 \%)$.

A análise descritiva e comparativa foi realizada comparando os grupos com menor dificuldade de fluência em leitura

Tabela 1. Ocorrência dos subperfis de alteração do PA ( $n=21)$

\begin{tabular}{|c|c|c|c|c|}
\hline \multirow[t]{2}{*}{ Subperfil } & \multicolumn{2}{|c|}{$\begin{array}{l}\text { Número de } \\
\text { ocorrências }\end{array}$} & \multicolumn{2}{|c|}{$\begin{array}{l}\text { Número de não } \\
\text { ocorrências }\end{array}$} \\
\hline & $\begin{array}{c}\text { Valor } \\
\text { absoluto }\end{array}$ & $\begin{array}{c}\text { Valor } \\
\text { relativo \% }\end{array}$ & $\begin{array}{c}\text { Valor } \\
\text { absoluto }\end{array}$ & $\begin{array}{c}\text { Valor } \\
\text { relativo \% }\end{array}$ \\
\hline Decodificação & 19 & 90,4 & 2 & 9,5 \\
\hline Integração & 19 & 90,4 & 2 & 9,5 \\
\hline Prosódia & 18 & 85,7 & 3 & 14,3 \\
\hline
\end{tabular}


(grupo A) e com maior dificuldade de fluência em leitura (grupo B). A divisão dos grupos foi realizada conforme os critérios já descritos na metodologia.

O teste exato de Fisher foi utilizado na comparação das variáveis categóricas apontando ausência de correlação estatisticamente significativa para gênero e idade. Posteriormente, a comparação das variáveis numéricas foi realizada através do teste de Mann-Whitney conforme mostra a Tabela 2.

Somente a variável memória sequencial verbal (MSV) foi estatisticamente significante $(\mathrm{p}=0,030)$ para o grupo com maior dificuldade de fluência em leitura (grupo B).

$\mathrm{Na}$ Tabela 3 é possível visualizar o desempenho dos participantes em relação aos escores de compreensão leitora e do ditado balanceado que constituíram variáveis entre os grupos A e B.

Para o grupo A, era esperado maior desempenho em compreensão leitora e ditado balanceado em função de ser o grupo com maior fluência em leitura. Porém, os participantes 2,11 e 13 obtiveram desempenho inferior a $50 \%$ de acertos na compreensão leitora, e os participantes 10, 20 e 21 apresentaram um número muito elevado de erros no ditado balanceado, número este, superior a 15 erros. Convém ressaltar que o padrão de normalidade para o final da $1^{\text {a }}$ série é de 10 erros. Assim, o número de erros esperado para a $2^{\mathrm{a}}$ série deveria ser inferior a 10 erros.

O mesmo ocorreu para o grupo B, os participantes 1, 3, 8 e 9 apresentaram bom desempenho na compreensão leitora com acertos superiores a $50 \%$.

Este, por ser o grupo com menor fluência em leitura, seria
Tabela 2. Comparação dos testes de PA ao considerar os grupos com menor dificuldade de fluência em leitura (grupo A) e com maior dificuldade de fluência em leitura (grupo $B)(n=21)$

\begin{tabular}{lccc}
\hline Variável & Grupo A Média & Grupo B Média & Valor de $\mathrm{p}^{*}$ \\
\hline SSWEC & 48,33 & 60,42 & $\mathrm{p}=0,154$ \\
SSWDC & 46,39 & 58,75 & $\mathrm{p}=0,199$ \\
TA & 2,44 & 3,00 & $\mathrm{p}=0,270$ \\
INV & 3,11 & 2,58 & $\mathrm{p}=0,880$ \\
IBOE & 44,44 & 57,50 & $\mathrm{p}=0,472$ \\
IBOD & 35,56 & 50,83 & $\mathrm{p}=0,352$ \\
ADD & 64,44 & 73,33 & $\mathrm{p}=0,690$ \\
ADE & 50,00 & 73,33 & $\mathrm{p}=0,115$ \\
PPSM & 73,28 & 72,52 & $\mathrm{p}=0,541$ \\
PPSN & 35,98 & 43,29 & $\mathrm{p}=0,474$ \\
LS & 3,67 & 3,50 & $\mathrm{p}=0,684$ \\
MSV & 0,00 & 0,42 & $\mathrm{p}=0,030^{* *}$ \\
MSNV & 2,67 & 2,92 & $\mathrm{p}=0,159$ \\
\hline
\end{tabular}

Legenda: SSWEC = teste SSW esquerda competitiva; SSWDC = teste SSW direita competitiva; $T A$ = tipo $A$; INV = inversões; IBOE = integração binaural orelha esquerda; IBOD = integração binaural orelha direita; $A D D=$ atenção direcionada à direita; $\mathrm{ADE}=$ atenção direcionada à esquerda; $\mathrm{PPSM}=$ padrão de freqüência condição murmurando; PPSN = padrão de freqüência condição nomeando; LS = localização sonora; MSV = memória sequencial verbal; MSNV = memória sequencial não-verbal.

* Valor p referente ao teste de Mann-Whitney para comparação das variáveis entre os grupos de dificuldade de aprendizagem

** Diferença significante $(p \leq 0,05)$

Tabela 3. Distribuição dos participantes em relação aos resultados da compreensão leitora (CL) e o ditado balanceado ( $n=21$ )

\begin{tabular}{|c|c|c|c|c|c|c|c|}
\hline \multicolumn{2}{|c|}{ Participantes } & \multirow[t]{2}{*}{ CL \% acertos } & \multicolumn{5}{|c|}{ Ditado balanceado } \\
\hline & & & CFG & RA & $\mathrm{RC}$ & ETF & Total de erros \\
\hline \multirow[t]{9}{*}{ Grupo A } & $\mathrm{P} 2$ & 20 & 3 & 3 & 5 & 2 & 12 \\
\hline & P6 & 70 & 0 & 3 & 7 & 3 & 13 \\
\hline & P10 & 60 & 10 & 5 & 9 & 2 & 24 \\
\hline & $\mathrm{P} 11$ & 20 & 42 & 5 & 2 & 5 & 53 \\
\hline & P13 & 40 & 0 & 1 & 4 & 1 & 6 \\
\hline & P18 & 70 & 5 & 3 & 6 & 0 & 14 \\
\hline & P19 & 60 & 6 & 2 & 6 & 0 & 14 \\
\hline & P20 & 80 & 42 & 3 & 0 & 2 & 47 \\
\hline & $\mathrm{P} 21$ & 60 & 9 & 5 & 5 & 0 & 19 \\
\hline \multirow[t]{12}{*}{ Grupo B } & $\mathrm{P} 1$ & 70 & 8 & 4 & 10 & 1 & 23 \\
\hline & P3 & 60 & 24 & 4 & 7 & 4 & 39 \\
\hline & $\mathrm{P} 4$ & 20 & 23 & 4 & 8 & 3 & 40 \\
\hline & P5 & 30 & 31 & 3 & 7 & 4 & 45 \\
\hline & $\mathrm{P} 7$ & 50 & 33 & 8 & 7 & 3 & 50 \\
\hline & P8 & 70 & 36 & 8 & 6 & 5 & 53 \\
\hline & P9 & 80 & 6 & 5 & 7 & 2 & 17 \\
\hline & P12 & 50 & 6 & 4 & 10 & 3 & 23 \\
\hline & P14 & 60 & 20 & 7 & 10 & 5 & 42 \\
\hline & P15 & 50 & 18 & 6 & 6 & 1 & 31 \\
\hline & P16 & 30 & 28 & 4 & 3 & 6 & 41 \\
\hline & P17 & 30 & 14 & 8 & 3 & 4 & 29 \\
\hline
\end{tabular}

Legenda: $\mathrm{CL}$ = compreensão leitora; $\mathrm{CFG}$ = conversor fonema grafema; $\mathrm{RA}$ = regras arbitrárias; $\mathrm{RC}$ = regras contextuais; $\mathrm{ETF}=$ erros transcrição da fala 
esperado que o desempenho em compreensão leitora fosse inferior ao grupo A.

\section{DISCUSSÃO}

A partir dos dados obtidos nesta pesquisa observou-se que não ocorreu diferença estatisticamente significante em relação ao gênero e idade. Porém, pesquisas destacam a prevalência de meninos com distúrbio de linguagem e aprendizagem ${ }^{(11,21)}$.

Nesta pesquisa a amostra foi constituída de 14 meninos $(66,7 \%)$ e sete meninas $(33,3 \%)$, com idades variando entre sete e 11 anos. Os escolares que apresentaram repetência não foram excluídos do estudo. Assim, considerando que as crianças ingressam na primeira série aos sete anos, segunda série aos oito anos, na terceira série aos nove e assim por diante, os participantes com nove anos repetiram a primeira ou a segunda série apenas uma vez. Já as crianças com 11 anos repetiram a primeira ou a segunda série mais de uma vez, sendo consideradas em idade avançada para a segunda série. Tais crianças podem, além de apresentar dificuldades de aprendizagem, evidenciar transtornos cognitivos influenciando a análise dos resultados. Convém ressaltar que os participantes desta pesquisa não foram submetidos à avaliação cognitiva por não haver profissional habilitado para tal no local da coleta de dados.

Estudos já realizados demonstraram que o desempenho nos testes de processamento auditivo aumenta com a idade, sendo considerada a faixa etária dos nove anos como um dos principais $\operatorname{marcos}^{(3,7-8)}$. Tais pesquisas foram realizadas entre participantes com e sem dificuldades de aprendizagem, destacando a referida melhora nos testes no grupo sem queixas de dificuldades de aprendizagem. Na pesquisa realizada não foi observada tal melhora nos testes de processamento auditivo.

Neste estudo os subperfis de maior ocorrência foram: decodificação e integração, ocorrendo em 19 dos 21 participantes independentemente do grupo em questão e o subperfil denominado prosódia ocorreu em 18 participantes.

O subperfil de decodificação é um dos mais comuns de acordo com o estudo realizado que avaliou 15 crianças através dos testes SSW e de compreensão leitora, sendo que, dos 11 que tiveram alteração do processamento auditivo oito apresentaram alteração do subperfil de decodificação ${ }^{(3)}$. Subperfil, este, que é responsável pela discriminação dos sons ${ }^{(2)}$.

Neste estudo com 21 participantes, 19 também apresentaram alteração no subperfil de integração. Outra pesquisa realizada encontrou alteração em quatro participantes dos 11 que apresentaram alteração de processamento auditivo ${ }^{(22)}$.

Em função da alta ocorrência em todos os subperfis primários estudados é possível questionar o diagnóstico de disfunção primária do processamento auditivo. Tais alterações podem decorrer de um atraso maturacional das estruturas inter-hemisféricas do córtex auditivo primário que interferem no desempenho das habilidades auditivas que são fundamentais para a aprendizagem da leitura e da escrita ${ }^{(14)}$. Tal atraso interfere de forma significativa no desempenho dos testes de processamento auditivo $^{(7)}$.

Ao comparar o grupo com maior fluência em leitura (grupo A) com o de menor fluência (grupo B), a única variável que se destacou foi a memória sequencial verbal (MSV), estatisticamente significativa para o grupo B. Este resultado condiz com a literatura como no estudo em que analisaram crianças com dificuldade de leitura e escrita com a avaliação simplificada do processamento auditivo ${ }^{(9)}$. Todas as crianças apresentaram alteração no teste da memória sequencial verbal com quatro sílabas.

Outra pesquisa foi realizada com crianças que apresentavam alterações no desenvolvimento da linguagem comparadas a um grupo controle sem tais alterações e observaram que o grupo com alterações na linguagem apresentava desempenho inferior nas provas de memória sequencial verbal e não verbal $^{(22)}$. O baixo índice de acerto em tais testes mostra a relação existente entre a aprendizagem e a memória de curto prazo. Tal memória é muito importante para a leitura e escrita, pois o conteúdo lido pelo leitor deve ser mantido na memória de curto prazo para que o mesmo acesse o sentido ${ }^{(9)}$.

A prova de memória sequencial verbal integra a avaliação simplificada do processamento auditivo cuja realização não requer o uso de audiômetro. Assim, professores e outros profissionais que trabalham com crianças com dificuldades de aprendizagem deveriam conhecer a importância desta prova ao observar desempenho abaixo do esperado e realizar encaminhamento à avaliação fonoaudiológica.

Nesta pesquisa o desempenho dos participantes em relação à compreensão leitora e escrita foi variável entre os grupos A (maior fluência em leitura) e B (menor fluência em leitura). Um estudo realizado mostrou que os erros na compreensão leitora aumentaram conforme aumentava as dificuldades na escrita $^{(12)}$. Tal resultado não foi corroborado nesta pesquisa, na qual não foi observada tal relação, pelo fato do tamanho da amostra $(n=21)$ e talvez, por incluir os alunos com repetência escolar.

O transtorno do processamento auditivo e as dificuldades de aprendizagem quando detectados precocemente, permitem a adequada orientação aos pais, facilitam a conduta de professores no processo de aprendizado e possibilitam o encaminhamento do aluno à avaliação fonoaudiológica clínica quando necessário. Deste modo, reforça-se a participação do fonoaudiólogo junto à equipe profissional que atua nas escolas não apenas no que se refere à orientação e ao encaminhamento, mas, também, fornecendo conhecimentos específicos à comunidade escolar como a possibilidade de tratamento fonoaudiológico para as dificuldades de aprendizagem e para o transtorno do processamento auditivo, pois inúmeros trabalhos mostram a eficácia de tais treinamentos ${ }^{(23-26)}$.

Maiores pesquisas devem ser realizadas com um número maior de participantes comparando esta população a crianças sem alteração de aprendizagem no que se refere ao processamento temporal incluindo a utilização de outros testes como RGDT e GIM ${ }^{(27-28)}$.

\section{CONCLUSÃO}

O presente estudo possibilitou a identificação da memória sequencial verbal como aspecto relevante ao relacionar os escores dos testes de processamento auditivo com as dificuldades de aprendizagem evidenciadas pela menor fluência em leitura. Várias pesquisas já apontaram que as dificuldades em 
memória sequencial verbal estão relacionadas aos distúrbios de comunicação e aprendizagem e sua dependência com a memória de curto prazo. Dessa forma, estudos com o processamento temporal devem ser realizados com um número maior de participantes, assim como a inclusão de grupo controle, delineamento inicial que não foi possível realizar.

Nesta pesquisa todos os participantes apresentaram trans- torno do processamento auditivo em quase todos os subperfis primários. Em função disso, questiona-se tal diagnóstico e formula-se a hipótese de atraso maturacional.

Convém ressaltar a importância do trabalho fonoaudiológico nas escolas objetivando orientar pais e professores quanto aos aspectos apontados, para fins de prevenção de um modo geral e encaminhamento dos casos verificados.

\begin{abstract}
Purpose: To clarify the relationship between learning difficulties and auditory processing disorder in second grade students. Methods: Based on the application of reading tests, the students of a second grade class of an elementary school were classified into two groups, according to their reading fluency: a group with better fluency (group A) and another with less fluency (group B). A between-group analysis of the auditory processing tests was carried out. Results: All participants presented learning difficulties and auditory processing disorder in almost every primary subprofiles. It was observed that the verbal sequential memory abilities of the less fluent group (group B) was significantly better $(\mathrm{p}=0,030)$. Conclusion: The diagnosis of primary auditory processing disorder is questioned, and it is emphasized the importance of stimulating verbal sequential memory to the learning of reading and writing abilities. In light of the results obtained, further researches are needed in order to study this variable and its relationship to temporal auditory processing.
\end{abstract}

Keywords: Auditory perception; Auditory perceptual disorders; Child; Reading; Learning; Developmental disabilities; Hearing disorders

\title{
REFERÊNCIAS
}

1. Alvarez AMMA, Caetano AL, Nastas SS. Processamento auditivo central. O que é isto? Fono Atual. 1997;1(1):17-8.

2. Jacob LCB, Alvarenga KF, Zeigelboim BS. Avaliação audiológica do sistema nervoso auditivo central. Arq Int Otorrinolaringol [Internet]. 2000;4(4): 144-51. [cited 2008 Jan 18]. Disponível em: http://www. arquivosdeorl.org.br/conteudo/acervo_port.asp?id=136.

3. Costa MID. Processamento auditivo central e compreensão leitora. Letras Hoje. 2003;39(3):179-97.

4. Neville HJ, Bavalier D. Specificity and plasticity in neurocognitive development in humans. In: Gazzaniga MS, editor. The new cognitive neurosciences. 2nd ed. Cambridge, Mass; London: MIT; 2000. p. 8398.

5. Manrique M, Cervera-Paz FJ, Huarte A, Perez N, Molina M, GarcíaTapia R. Cerebral auditory plasticity and cochlear implants. Int J Pediatr Otorhinolaryngol. 1999;49 Suppl 1:S193-7.

6. Robinson K. Implications of developmental plasticity for the language acquisition of deaf children with cochlear implants. Int J Pediatr Otorhinolayngol. 1998;46(1-2):71-80. Review.

7. Neves IF, Schochat E. Maturação do processamento auditivo em crianças com e sem dificuldades escolares. Pró-Fono. 2005;17(3):311-20.

8. Simon LF, Rossi AG. Triagem do processamento auditivo em escolares de 8 a 10 anos. Psicol Esc Educ. 2006;10(2):293-304.

9. Furbeta TDC, de Felippe AC. Avaliação simplificada do processamento auditivo e dificuldades de leitura-escrita. Pró-Fono. 2005;17(1):11-8.

10. Smith C, Strick L. Dificuldades de aprendizagem de A a Z: um guia completo para pais e educadores. Porto Alegre, RS: Artmed; 2001.

11. Moojen S. Caracterizando os transtornos de aprendizagem. In: Bassols AMS, Santis MFB, Sukiennik PB, Cristóvão PW, Fortes $\mathrm{SD}$, organizadores. Saúde mental na escola 1: uma abordagem multidisciplinar. Porto Alegre: Mediação; 2003. p. 98-110.

12. Zucoloto KA, Sisto FF. Dificuldades de aprendizagem em escrita e compreensão em leitura. Interação Psicol. 2002;6(2):157-66.

13. Alvarez AMMA, Caetano AL, Nastas SS. Processamento auditivo central: avaliação e diagnóstico. Fono Atual. 1997;1(1):34-6.

14. Bellis TJ. Assessment and management of central auditory processing disorders in the educational setting: from science to practice. $2 \mathrm{a}$ ed. New York: Thomson Delmar Learning; 2003.

15. Alvarez AMMA, Zaidan E, Balen SA, Garcia AP. Disfunção não verbal. Acta AWHO. 2000;19(1):49-55.

16. Jerger J. Clinical Experience with Impedance Audiometry. Arch Otolaryngol. 1970;92(4):311-24.

17. Saraiva RA, Moojen SMP, Munarski R. Avaliação da compreensão leitora textos expositivos. São Paulo: Casa do Psicólogo; 2005.

18. Salles JF, Parente MAMP. Funções neuropsicológicas em crianças com dificuldades de leitura e escrita. Psicol Teor Pesqui. 2006;22(2):153-62.

19. Auditec. Evaluation manual of pitch pattern sequence and duration pattern sequence. Missouri, USA: Auditec; 1997.

20. Pereira LD, Schochat E. Processamento auditivo central: manual de avaliação. São Paulo: Lovise; 1997.

21. Corona AP, Pereira LD, Ferrite S, Rossi AG. Memória sequencial verbal de três e quatro sílabas em escolares. Pró-Fono. 2005;17(1):27-36.

22. Befi-Lopes DM, Pinheiro IM, Rodrigues A. A memória seqüencial verbal e não-verbal nas alterações do desenvolvimento da linguagem. J Bras Fonoaudiol. 2002;3(11):148-54.

23. Tallal P. Improving language and literacy is a matter of time. Nat Rev Neurosci. 2004;5(9):721-8

24. Kozlowski L, Wiemes GMR, Magni C, Silva ALG. A efetividade do treinamento auditivo na desordem do processamento auditivo central: estudo de caso. Rev Bras Otorrinolaringol. 2004;70(3):427-32.

25. Russo NM, Nicol TG, Zecker SG, Hayes EA, Kraus N. Auditory training improves neural timing in the human brainstem. Behav Brain Res. 2005;156(1):95-103.

26. Zalcman TE, Schochat E. A eficácia do treinamento auditivo formal em indivíduos com transtorno de processamento auditivo. Rev Soc Bras Fonoaudiol. 2007;12(4):310-4.

27. Lister JJ, Roberts RA, Shackelford J, Rogers CL. An adaptive clinical test of temporal resolution. Am J Audiol. 2006;15(2):133-40.

28. Zaidan E, Garcia AP, Tedesco MLF, Baran JA. Desempenho de adultos jovens normais em dois testes de resolução temporal. Pró-Fono. 2008;20(1):19-24. 